\title{
Canabinoides y endocanabinoides como herramienta para el desarrollo de posibles antineoplásicos en caninos. Una revisión
}

\author{
A review of cannabinoids and endocannabinoids as a tool for \\ developing possible anti-neoplastic agents in dogs
}

\author{
Canabinóides e endocanabinóides como ferramenta para o \\ desenvolvimento de possíveis medicamentos antineoplásicos em cães. Uma \\ revisão
}

Fabio Mayorga-Niño ${ }^{1}$, Giovanny Torres-Vidales ${ }^{2}$

Grupo de investigación en Química Medicinal, Profesor asociado Escuela de Medicina, Facultad de Ciencias de la Salud, UPTC. Grupo de investigación en Medicina Veterinaria y Zootecnia, Profesor Asistente Escuela de Medicina Veterinaria y Zootecnia, Facultad de Ciencias Agropecuarias, UPTC.

Email: fabio.mayorga@uptc.edu.co

Recibido: Marzo 14 de 2013. $\quad$ Aceptado:

\begin{abstract}
Resumen
El tratamiento de los crecimientos neoplásicos malignos en caninos, incluye diferentes alternativas, entre las cuales se encuentra la quimioterapia. Sin embargo, los fármacos tradicionalmente utilizados presentan altos niveles de toxicidad, debido a su baja selectividad sobre células cancerosas. Los principales efectos adversos de estos compuestos son trastornos gastrointestinales, depresión de la medula ósea, alteraciones de la conducta, alopecia y un potencial carcinogénico, entre otros. En la actualidad, diferentes grupos de investigación en el mundo han estado dedicados al desarrollo de nuevas moléculas derivadas de canabinoides y/o endocanabinoides, basados en la reconocida actividad antineoplásica del tetrahidrocanabinol y de la anandamida. Los ensayos hasta ahora reportados permiten concluir que dichos compuestos ofrecen una mayor selectividad de acción sobre las células enfermas, lo cual se traduce en un menor abanico de riesgos tóxicos. Por otra parte, se ha demostrado que los tratamientos farmacológicos en cualquier patología deberían ceñirse a los ritmos biológicos con el fin de obtener una mejor respuesta terapéutica y un menor grado de efectos colaterales.
\end{abstract}

El objetivo de este artículo es dar a conocer los beneficios de un futuro tratamiento antineoplásico inspirado en el comportamiento de los canabinoides y endocanabinoides en la evolución de las enfermedades neoplásicas.

Palabras clave: Crecimiento neoplásico, canabinoides, endocanabinoides, cronofarmacología.

\begin{abstract}
There are several alternatives for treating malign neoplastic growths in dogs, including chemotherapy; however, traditionally used drugs have high levels of toxicity due to their low selectivity regarding cancer cells. These compounds' main adverse effects involve gastrointestinal disorders, bone marrow suppression (myelotoxicity),
\end{abstract}


behavioral alterations, alopecia and carcinogenic potential. Research groups around the world are currently working on developing new drugs from cannabinoids and/or endocannabinoids-derived molecules, based on tetrahydrocannabinol and anandamide's known anti-neoplastic activity. Trials reported to date have led to concluding that such compounds offer greater selectivity regarding action on unhealthy cells, thereby representing a lower range of toxic risks. It has been shown that pharmacological treatment regarding any disease should adhere to biological rhythms to obtain a better therapeutic response and incur fewer collateral effects.

This article was thus aimed at ascertaining the benefits of future antineoplastic treatment inspired by the behavior of cannabinoids and endocannabinoids in the evolution of neoplastic diseases.

Key words: Neoplastic growth, cannabinoid, endocannabinoid, chronopharmacology.

\section{Resumo}

O tratamento de tumores malignos em cães, inclui diversas alternativas, entre as quais está a quimioterapia. Embora, as drogas convencionalmente usadas têm níveis elevados de toxicidade, devido à sua baixa selectividade sobre células cancerosas. Os principais efeitos adversos desses compostos são distúrbios gastrointestinais, depressão da medula óssea, distúrbios comportamentais, alopecia e um potencial carcinogénico, entre outras. Atualmente, diferentes grupos de pesquisa no mundo têm se dedicado ao desenvolvimento de novas moléculas derivadas de canabinóides e/ou endocanabinóides, baseados na conhecida atividade antineoplásica do tetrahidrocanabinol e da anandamida. Os experimentos relatados até agora levam à conclusão de que estes compostos oferecem uma maior seletividade de ação nas células doentes, o que resulta num menor intervalo de riscos tóxicos. Além disso, foi demonstrado que os tratamentos farmacológicos em qualquer doença deveríam estar em conformidade com os ritmos biológicos, a fim de obter uma melhor resposta terapêutica e um menor grau de efeitos colaterais.

O objetivo deste artigo é apresentar os benefícios de um futuro tratamento antineoplásico inspirado no comportamento dos canabinóides e endocannabinoides na evolução das doenças neoplásicas.

Palavras Chave: Crescimento neoplásico, Canabinóides, Endocanabinóides, Cronofarmacología.

\section{Introducción}

El crecimiento neoplásico se define como una proliferación autónoma y progresiva de las células propias de un individuo, que en la mayoría de los casos se correlaciona con la aparición de una masa o tumor. Este tipo de crecimientos se puede desarrollar en cualquier especie animal; sin embargo, se ha observado que la mayor casuística se presenta en caninos (Wihtrow and Vail, 2009)

En la práctica clínica de pequeños animales, y en especial para la especie canina, es frecuente el diagnóstico de crecimientos neoplásicos en diferentes sistemas orgánicos. Estos crecimientos pueden ser de diferente origen, dentro de los que se incluyen mesenquimales, epiteliales, de células redondas y de células productoras de pigmentos (Meuten, 2002). En cuanto al comportamiento, independientemente del origen, las neoplasias se clasifican como benignos o malignos, y éstas últimas pueden ser localizadas o difusas, como es el caso de la leucemia. Las neoplasias malignas locali- zadas, eventualmente pueden expandirse a diferentes tejidos, proceso que se conoce como metástasis (Paolini and Khanna, 2008).

En cuanto a las alternativas de tratamiento, estas pueden ser de tipo quirúrgico, físico (radioterapia) y químico (quimioterapia). La finalidad de la terapia farmacológica sistémica es controlar el crecimiento neoplásico en el foco primario y en sitios de metástasis (Bracho, 2011). En la actualidad, diferentes grupos de investigación en el mundo han venido demostrando las bondades del uso de canabinoides, endocanabinoides o sus análogos en el tratamiento del cáncer (Torres et al., 2011; Donadelli et al., 2011; Velasco, Sánchez and Guzmán, 2012). Sin embargo, son bien conocidos los efectos psicotrópicos que estos compuestos producen en el individuo (Rodríguez y Carrillo, 2005), a los cuales no son ajenos los animales.

Asimismo, la medicina moderna tiende a incorporar el concepto de cronofarmacología en el tratamiento de las diferentes patologías, entre ellas el cáncer, con 
el propósito de incrementar sus acciones terapéuticas, disminuyendo de forma importante sus efectos tóxicos (Ohdo, 2010).

El propósito de esta revisión es presentar el uso potencial de canabinoides y endocanabinoides como una alternativa que a futuro podrá constituir un método efectivo y seguro en el tratamiento del crecimiento neoplásico maligno en caninos.

Diferentes autores han reportado la actividad antineoplásica de canabinoides y endocanabinoides (Sarfaraz et al., 2008; Fowler et al., 2010; Cudaback et al., 2010).

\section{Principales fármacos utilizados en cáncer canino}

La quimioterapia es el uso de medicamentos para inhibir el crecimiento celular y, en medicina humana y veterinaria, es una alternativa para el tratamiento del cáncer.

Los fármacos que se utilizan en el tratamiento contra el cáncer son agentes citotóxicos que actúan principalmente sobre células que se encuentran en rápida división. Una dificultad que presenta la quimioterapia es la baja selectividad de los fármacos utilizados, ya que estos no discriminan entre células sanas de alta proliferación y células neoplásicas, las cuales presentan esta misma característica (Aurrecochea, 2008).

Los principales efectos adversos que se pueden presentar en mayor o menor medida con el uso de cualquiera de los agentes citotóxicos son: mielosupresión, principalmente neutropenia y trombocitopenia; anemia, la cual es menos frecuente por la larga vida media de los eritrocitos; nauseas, vómitos diarreas y anorexia; infertilidad y teratogénesis; cardiotoxicidad, nefrotoxicidad $y$, a su vez, todos son potencialmente carcinogénicos (Adams, 2001; Kukanich, 2012).

En la actualidad, los fármacos más utilizados en medicina veterinaria para el tratamiento del cáncer son:

\section{Agentes alquilantes}

Son sustancias que tienen capacidad electrofílica, propiedad que les permite introducir grupos alquilo a residuos guanina del ADN, interfiriendo de esta manera con los procesos de replicación y transcripción, lo cual altera la funcionalidad de los ácidos nucleicos y la progresión del ciclo celular.

Los efectos tóxicos comunes para todos los agentes alquilantes incluyen mielosupresión, toxicidad gastrointestinal manifestada en forma de náuseas, vómito, diarrea y daño de la mucosa del aparato digestivo. Toxicidad gonadal que conlleva a mala calidad seminal con oligospermia o aspermia, debido a depleción de las células germinales. Toxicidad pulmonar, ya que puede ocurrir neumonía intersticial y fibrosis. Estos agentes también son responsables del cuadro de alopecia que pueden presentar los pacientes durante el tratamiento (Aurrecochea, 2008). Dentro de los agentes alquilantes se utilizan compuestos como: mostazas nitrogenadas, nitrosoureas, entre otros.

Mostazas nitrogenadas. Entre estos agentes se encuentran, por ejemplo, ciclofosfamida, ifosfamida, melfalán y clorambucil, que son utilizados en el tratamiento tumores malignos de glándula mamaria, linfosarcomas y mastocitomas indiferenciados (Souza et al., 2009).

Las reacciones adversas específicas para este grupo de antineoplásicos incluyen cistitis hemorrágica estéril, acción teratogénica, leucemia y nefrotoxicidad (Maddison et al., 2004).

Nitrosoúreas. Son compuestos muy liposolubles, entre los que se encuentran carmustina, lomustina, semustina y nemustina. Todos traspasan con facilidad la barrera hematoencefálica, por lo tanto son utilizados en neoplasias cerebrales; otros usos incluyen el tratamiento de linfosarcomas multicéntricos y epiteliotropos y mastocitomas del tracto gastrointestinal (Van Meervenne et al., 2012).

Dentro de las reacciones adversas más comunes se encuentran, además de las descritas para los agentes alquilantes en general, hemiplejía, convulsiones, cefalea, confusión, edema cerebral, afasia, somnolencia, infección en el tracto urinario, tromboflebitis profunda y hepatotoxicidad (Moore and Kitchell, 2003).

\section{Cisplatino y sus derivados}

Este grupo incluye cisplatino, carboplatino y oxaliplatino. Tales productos reaccionan con los residuos de la posición N7 de la guanina y adenina, para formar una amplia variedad de aductos mono, o bifuncionales. Dichos aductos impiden ciertos procesos celulares que requieren la reparación de ambas cadenas de ADN. Por otra parte, la unión a proteínas citoplasmáticas o nucleares puede tener efectos citotóxicos (Vail et al., 2007).

En pequeños animales son usados para el tratamiento de carcinomas escamocelulares, mesoteliomas, linfosarcomas y cáncer de pulmón, entre otros. Sus efectos adversos incluyen nefrotoxicidad, neurotoxicidad, náuseas, vómito, ototoxicidad, alopecia y desequilibrio electrolítico (Calvelo, 2013). 


\section{Antimetabolitos}

A este grupo pertenecen el metotrexate y el 5-fluorouracilo, entre otros. Estos son utilizados en el tratamiento del cáncer de glándula mamaria, linfomas y osteosarcomas. Los antimetabolitos compiten con los nucleósidos fisiológicos; tienen un triple mecanismo de acción a través de la incorporación y alteración de las macromoléculas de ADN y ARN, por interferencia con las enzimas implicadas en la síntesis de ácidos nucleicos o por modificación del metabolismo de los nucleósidos fisiológicos.

Los efectos adversos para este grupo incluyen anemia, hepatotoxicidad, náuseas, vómito y cambios en la pigmentación de la piel (Maddison et al., 2004).

\section{Antibióticos antitumorales}

En este grupo se encuentran las antraciclinas, que incluyen la doxorrubicina y daunomicina. Tienen efecto antitumoral, ya que actúan en la fase $\mathrm{S}$ del ciclo celular, inhibiendo el ADN y ARN. Dicho proceso está mediado por la inhibición de la topoisomerasa II y la generación de radicales libres. Los antibióticos antitumorales son utilizados en el tratamiento del hemangiosarcomas, liposarcomas infiltrativos, linfosarcomas y tumor venéreo transmisible. Los efectos adversos de las antraciclinas incluyen mielosupresión, toxicidad cardiaca, reacción alérgica local y trastornos gastrointestinales y pulmonares (Elliot et al., 2013; Reagan et al., 2013).

\section{Alcaloides de la vinca}

Este grupo incluye vincristina, vinblastina, vindesina y vinorelbina. Estas sustancias son ciclo específicas, ya que actúan interfiriendo en la polimerización de la tubulina, alterando de esta forma la función de los microtúbulos que forman el huso mitótico, desencadenando así la muerte celular. Los alcaloides de la vinca son utilizados en la clínica de pequeños animales para el tratamiento de carcinomas mamarios, carcinomas nasales, tumor venéreo transmisible, nefroblastomas, adenocarcinomas pancreáticos, mastocitomas y leucemias. Dentro de los efectos adversos para este grupos se incluye neutropenia severa, signos de neurotoxicidad por dosis acumulativas, pérdida de fuerza muscular e irritación local fuerte cuando se presenta extravasación (Herrera et al, 2006).

\section{El sistema endocanabinoide}

En las últimas dos décadas se ha identificado, tanto en animales como en seres humanos, el Ilamado sistema endocanabionoide, el cual está constituido por los receptores canabinoides, los ligandos endógenos y las enzimas responsables de su formación y destrucción (Pertwee, 2008; Velasco, Sánchez and Guzmán, 2012).

\section{Receptores}

Hasta 1988 se pensaba que la marihuana ejercía sus efectos de una manera independiente de receptores. Para esta época se descubrieron receptores sobre los cuales se confirmó la acción del $\Delta^{9}$-THC, en el cerebro de porcinos. A aquellos receptores se les denominó CB1. Más adelante se identificaron otros receptores similares en zonas corporales periféricas, a los que se denominó CB2 (Munro et al., 1993; Guzmán, 2003; Malfitano et al., 2011). Posteriormente, en 1990, Matsuda y colaboradores reportaron la caracterización del receptor CB1 y en 1993, Munro y colaboradores informaron de la plena identificación del receptor CB2.

Se ha establecido que los receptores CB1 se localizan principalmente en el sistema nervioso central, determinándose en forma bastante detallada la distribución de estos receptores en los cerebros de algunos animales y en seres humanos (Herkenham et al., 1990; Mailleux and Vanderhaeghen, 1992; Tsou et al 1998; Smith et al., 2010). Así mismo, se encuentran en menor concentración en algunos tejidos periféricos, tales como el bazo, el corazón, la próstata, los ovarios, el útero y, a nivel presináptico, en terminales nerviosas (Galiegue et al., 1995; Ishac et al., 1996; Pertwee et al., 2010).

Los receptores CB2, por su parte, se localizan principalmente en el bazo, amígdalas y sistema inmune. Los efectos inmunosupresores de la marihuana pueden explicarse por la presencia de receptores CB2 en estos tejidos (Mayorga y Cárdenas, 2009; Atwood and Mackie, 2010).

Los principales mecanismos intracelulares en los que están implicados los receptores CB1 incluyen inhibición de la adenilato ciclasa, regulación de diferentes canales iónicos y la activación de la vía de las MAP quinasas (Howlett, 1998). La estimulación de los receptores canabinoides induce una inhibición de los canales de calcio y un aumento de la conductancia del potasio. El efecto combinado sobre ambos tipos de canales parece ser la base de la inhibición de la liberación de neurotransmisores (Bouaboula et al., 1995; Patcher et al., 2006; Katona and Freund, 2008).

Además de los tipos de receptores ya comentados, los canabinoides y endocanabinoides pueden actuar so- 
bre receptores vanilloides (Grotenherm, 2004; Marini et al., 2009).

\section{Endocanabinoides}

Los ligandos de los receptores CB1 y CB2, así como de los vanilloides inicialmente conocidos, eran los canabinoides derivados de la Cannabis sativa, principalmente el $\Delta^{9}$-THC. Cuando en 1988 se descubrieron primero los receptores CB1 y luego los CB2, se pensó que la presencia de estos receptores suponía la existencia de ligandos endógenos, específicos de tales receptores. Fue esto lo que motivó a los científicos a investigar en la búsqueda e identificación de estos compuestos hasta entonces desconocidos. Así, en 1992, Devane y colaboradores reportaron el aislamiento de un nuevo constituyente cerebral, encontrado por ellos en el cerebro de porcinos, pero que luego fue detectado en humanos y muchos otros animales. A este nuevo compuesto lo llamaron anandamida. Mechoulam, en 1995, informó de la presencia de otro compuesto de alto interés: el 2-araquidonoilglicerol.

Se ha demostrado que los endocanabinoides, especialmente la anandamida, están implicados en múltiples procesos biológicos entre los cuales están: desarrollo neuronal, acción neuromoduladora, metabolismo, funciones cardiovascular, respiratoria, endocrina, sexual, sanguínea, así como en procesos inflamatorios, analgesia, respuesta inmune y crecimiento neoplásiCo, entre otros (Bosier et al., 2010; Jenny et al., 2010; Guindon and Hohmann, 2011)

Químicamente la anandamida es la etanolamida del ácido araquidónico, lo cual habla claramente de su posible relación con las prostaglandinas, leucotrienos, tromboxanos y, en fin, con aquellos metabolitos que se desprenden de la cascada del ácido araquidónico y que están implicados en importantes procesos como la inflamación, función sanguínea, contracción de músculo liso, etc. La síntesis de la anandamida, que es el canabinoide endógeno más estudiado hasta la fecha, se produce mediante la hidrólisis de la $\mathrm{N}$-araquidonilfosfatidiletanolamina, un precursor fosfo-lipídico. Este proceso se lleva a cabo catalizado por una fosfolipasa D (Giuffrida et al., 1999). Esta hidrólisis ocurre en el momento en que el organismo requiera la liberación de la anandamida. Esta liberación se hace de una manera dependiente de canales de calcio; sin embargo, existe evidencia de que receptores acoplados a proteínas G pueden disparar este proceso, tal como sucede con los receptores dopaminérgicos $D_{2}$ (Liu et al., 2008; Jenny et al; 2010). La vida media de la anandamida es muy corta, lo cual hace difícil la tarea de su aislamiento. La enzima en- cargada de realizar la hidrólisis es la amidohidrolasa de ácido graso (FAAH) (Mallat et al., 2011).

Además de la anandamida y del 2-araquidonoilglicerol, posteriormente se descubrieron otros endocanabinoides que ofrecen interesantes perspectivas farmacológicas: noladín éter, que activa receptores CB1 (Porter et al., 2002); la virodhamina, es el éster del ácido araquidónico y la etanolamina, que actúa sobre receptores CB1 (Huang et al., 2002) y, finalmente, el agonista vanilloide $\mathrm{N}$-araquidonoildopamina, que también exhibe afinidad por receptores canabinoides (Nelson et al., 2001).

Como ya se adelantó, existen enzimas responsables de la formación e hidrólisis de la anandamida, el endocanabinoide que ofrece mayor perspectiva e interés farmacológico. En la formación de la anandamida interviene una fosfolipasa $\mathrm{D}$, para permitir su liberación a partir de la $\mathrm{N}$-araquidonilfosfatidiletanolamina, y para su degradación actúa la FAAH (Mallat et al., 2011).

\section{Nuevas perspectivas farmacológicas para el tratamiento del cáncer en caninos}

Desde siglos atrás, la marihuana ha sido utilizada con propósitos medicinales y recreativos. En la actualidad, la medicina aprovecha los efectos antieméticos y analgésicos del tetrahidrocanabinol (THC), presente en la marihuana, como paliativo de algunos síntomas del cáncer y su tratamiento (Guzmán, 2003; Pertwee, 2009; Velasco et al., 2012).

Investigaciones recientes han demostrado que el THC es mucho más que un paliativo. En 1975 se conoció la actividad antineoplásica del THC (Munson et al., 1975; Guzmán, 2003; Sarfaraz et al., 2008), pese a que posteriormente se han reportado estudios que muestran que los canabinoides también pueden presentar efectos pro-proliferativos en cáncer, cuando se administran a dosis micromolares, ya que a estas concentraciones ejerce actividad antiapoptótica (Hart et al., 2004; Flygare and Sander, 2008; Guindon and Hohmann, 2011). En 1998, De Petrocellis, Bifulco, Di Marzo y col, así como Sánchez y colaboradores en 2006 se pronunciaron respecto a la importante acción inhibitoria de la anandamida sobre la proliferación de células de cáncer de seno humano, a través de la activación de receptores CB2; lo que a su vez detiene el ciclo celular en la transición G2-M, vía subregulación del gen $\mathrm{Cdc} 2$, induciendo de esta forma la muerte celular por apoptosis. Con base en este conocimiento en humanos, y dado que no existen estudios al respecto en caninos, podemos inferir que tal afirmación pudiera ser extrapolable a tal especie, dadas las similitudes 
etiológicas, histológicas y bioquímicas de esta patología entre dichas poblaciones; situación que deber comprobarse con futuras investigaciones. Bifulco y col. en 2001 estudiaron la influencia del sistema canabinoide en el control del crecimiento de tumores dependiente del oncogén ras. Bifulco y Di Marzo en 2002 convocaron a unir esfuerzos en la búsqueda de nuevas moléculas derivadas de endocanabinoides con el propósito de lograr un mejor control del cáncer. Guzmán insistió en 2003 en el potencial anticancerígeno de los canabinoides, y sobre esta base, también se deberá explorar la acción de los endocanabinoides. La inhibición de la angiogénesis tumoral por parte de los canabinoides fue propuesta por Jorcano y colaboradores en 2003 y, posteriormente, por Strieter en 2005. Nuevamente, Blázquez, Guzmán y colaboradores, en 2004, informaron que los canabinoides inhiben el factor de crecimiento endotelial en gliomas, como un importante aporte al entendimiento de la acción de estos compuestos sobre células cancerosas. El efecto positivo de los canabinoides en el control del cáncer de próstata fue reportado por Sarfaraz y colaboradores en 2005. Ese mismo año, Nithipatikon y su grupo de trabajo informaron de nuevos inhibidores de la hidrólisis del 2-araquidonoilglicerol como alternativa para el tratamiento de neoplasias malignas de próstata; mientras que Grimaldi, Di Marzo, Bifulco y colaboradores confirmaron en 2006 y Guindon and Hohmann en 2011, la interesante acción inhibitoria de la anandamida sobre células de cáncer de seno. De Morrow y col. reportaron en 2007 la acción de los endocanabinoides sobre el crecimiento del colangiocarcinoma; en abril del mismo año, Bifulco, Laezza, Gazzero y Pentimalli publicaron una interesante revisión acerca de los endocanabinoides como supresores emergentes de la angiogénesis y la invasión tumoral, y, como ya se ha mencionado, son muchos los investigadores que se han dedicado a estudiar los posibles éxitos de la terapia canabinoide en el tratamiento del cáncer.

\section{Posibles mecanismos de acción antineoplásica de canabinoides/endocannabinoides}

En la actualidad se han propuesto diferentes mecanismos de acción antineoplásica de los canabinoides y los endocanabinoides. Una de las formas que ha resultado útil para conseguir la acción antineoplásica de los endocanabinoides es el bloqueo de las enzimas que inactivan dichos compuestos. Se ha demostrado que los niveles de canabinoides endógenos, principalmente anandamida, resultan ser considerablemente más altos en células cancerosas y premalignas, que en tejidos normales (Ligresti et al., 2003). Basados en esto, se han hecho intentos por incrementar la concentración local de endocanabinoides en las células tumorales blo- queando su sistema de transporte o las enzimas que los inactivan, como la monoacilglicerol (MAGL) y la amidohidrolasa de ácido graso (FAAH). De esta manera, se han inducido los efectos antitumorales de las rutas de señalización de los receptores canabinoides (CB), en varios tipos de cáncer como los de tiroides, cerebro y próstata (Bifulco et al., 2004; Nithipatikon et al., 2004; Nithipatikon et al., 2005; De Lago et al., 2006; Endsley et al., 2007). El bloqueo del metabolismo de la anandamida puede resultar útil en el control del cáncer, ya que además de incrementar los niveles de este endocanabinoide, evita la generación de etanolamina (Matas et al., 2007). Sin embargo, se ha observado que las sustancias hasta ahora ensayadas para bloquear las enzimas que degradan la anandamida y el 2-araquidonoilglicerol, así como el inhibidor del transporte de anandamida VDM11, presentan algunos efectos colaterales diversos e inespecíficos (De Lago et al., 2006); lo cual motiva el diseño de nuevas moléculas con el propósito de conseguir una acción más específica y limpia sobre la degradación y transporte de los endocanabinoides. Esta estrategia convendría ensayarla paralelamente con consideraciones acerca de los ritmos biológicos celulares que pudieran alterar el comportamiento de las células cancerígenas (Goldbeter et al., 2007), así como el momento de mayor activación de las MAGL y FAAH, lo cual constituiría hasta ahora un criterio novedoso en la lucha por la búsqueda de nuevas herramientas contra el cáncer (Smolensky and Peppas, 2007).

Existen efectos directos e indirectos de los canabinoides sobre el cáncer que se pueden explicar a través de diferentes mecanismos de acción. Se sabe que los canabinoides exógenos pueden atacar directamente las células cancerosas a través de su unión a receptores CB. Aquello afecta las rutas de señalización celular, lo cual puede inducir la supresión del crecimiento celular, así como su muerte o inhibir la migración. Además, los canabinoides pueden actuar indirectamente, inhibiendo el proceso de la angiogénesis o por interferencia con el sistema inmune (Flygare and Sander, 2008). En tumores cerebrales, los canabinoides inducen la muerte celular porque estimulan la síntesis de ceramida de novo, resultando en apoptosis (Gómez del Pulgar et al., 2002; Gomez del Pulgar and De Ceballos, 2002; Flygare and Sander, 2008).

Dado que el cáncer de seno puede ser dependiente de hormonas como los estrógenos y la prolactina, cuando se da, los canabinoides inducen la supresión del crecimiento porque influyen en la subregulación de los receptores de prolactina, además de contrarrestar los efectos pro proliferativos de la prolactina misma (Flygare an Sadner, 2008; Guindon and Hohmann, 2011). 
Sin embargo, se ha demostrado que los canabinoides, además, inhiben el cáncer de seno por acción directa sobre receptores CB2 (Grimaldi et el., 2006).

En el cáncer de próstata incurren al menos tres factores sobre los cuales actúan los canabinoides: por una parte, en sus células se expresan niveles superiores de receptores CB1 y CB2 a las células normales de próstata; en segundo lugar, de forma similar a como ocurre con el cáncer de seno, en las células cancerosas de próstata también se expresan receptores de prolactina (Melck et al., 2000; Guindon and Hohmann, 2011). Finalmente, el receptor del factor de crecimiento endotelial, EGFR, encontrado en tales células. Los canabinoides actúan sobre los tres factores, controlando de esta manera el crecimiento de las células anormales. La acción sobre los EGFR resulta en una inhibición de la proliferación en el día 3 y en muerte celular masiva en el día 5 por apoptosis/necrosis (Mimeault et al., 2003). Además, se ha reportado que los canabinoides subregulan el receptor de andrógenos y el antígeno específico de próstata (Sarfaraz et al., 2006).

En cáncer de colon, también se expresan receptores CB1 y CB2, y se sabe que los canabinoides inducen apoptosis de estas células anormales a través de efectos mediados por receptores CB1 (Flygare and Sander, 2008). Otro mecanismo a través del cual actúan los canabinoides es la inhibición de la angiogénesis, bloqueando los factores proangiogénicos o por efecto directo sobre la formación de vasos in vivo. Esto ha sido especialmente estudiado en cáncer de piel, tiroides y gliomas, ya que se ha observado que los canabinoides inhiben la producción del factor de crecimiento endotelial vascular, VEGF (Portella et al., 2003; Blázquez et al., 2004), un factor proangiogénico. Actualmente se avanza en el entendimiento de si los canabinoides son realmente efectivos en condiciones como la leucemia mieloide aguda (AML), síndromes mielodisaplásicos o desórdenes mieloproliferativos (Flygare and Sander, 2008), ya que ha sido documentado que condiciones como la AML expresan receptores CB2 (Alberich et al., 2004)

Cuando se analiza el potencial terapéutico de los canabinoides en cáncer, deben considerarse los posibles efectos procancerígenos de estos compuestos, ya que existen algunos reportes que indican que compuestos como el THC y la anandamida pueden inducir activación del factor de crecimiento endotelial (VEGF) en líneas de células de cáncer, tales como cáncer de pulmón, carcinoma escamoso de piel, carcinoma de vejiga, glioblastoma, astrocitoma y cáncer de riñón vía activación de receptores CB2, cuando existen concentraciones micromolares de los canabinoides (Hart et al., 2004).
Por otra parte, puesto que los canabinoides pueden suprimir las respuestas mediadas por células y la respuesta humoral inmune, deben tenerse en cuenta los efectos sobre la inmunidad del tumor cuando se considere atacar el sistema canabinoide in vivo (Klein et al., 2003). La administración de canabinoides a ratones transplantados, con cáncer, carentes de receptores CB, puede promover el crecimiento del cáncer mediante la supresión de la respuesta anti-tumoral inmune. Se ha demostrado, además, que el antagonista del receptor canabinoide CB1, SR141716A, en bajas dosis, presenta efectos anticancerígenos en algunas líneas de cáncer, tales como cáncer de seno, de tiroides y carcinoma de colon (Sarnataro et al., 2006). Se ha postulado un mecanismo de acción antineoplásico diferente, de los endocannabinoides, particularmente de la anandamida, a través de receptores vanilloides (Maccarrone et al., 2000; Contassot et al., 2004). También se ha sugerido que la anandamida induce muerte celular de manera independiente del receptor, a través de su interacción con dominios de membrana ricos en colesterol. Lo mismo sucede con el colangiocarcinoma, en el que la anandamida induce apoptosis e inhibe la proliferación celular mediante esta interacción, a través de la acumulación de ceramida (De Morrow et al., 2007). Otro mecanismo observado a partir de estudios en animales de experimentación, implica la interacción de la ciclooxigenasa (COX) y la lipooxigenasa (LOX), lo cual se observó cuando la administración de metanandamida estimuló la proliferación celular de una manera independiente de receptores $\mathrm{CB}$, mediante la inducción de COX-2 (Gardner et al., 2003), mientras una dosis 10 veces menor redujo la metástasis de una manera dependiente del receptor CB1 (Portella et al., 2003).

Finalmente, existe una creciente evidencia de que los canabinoides pueden actuar de una manera selectiva sobre células cancerosas, ya que estas expresan con mayor facilidad receptores CB en comparación con las células sanas, lo cual hace que su acción sobre éstas últimas sea mínima. Lo anterior explica la menor sensibilidad de estas células a la acción de tales compuestos (Flygare and Sander, 2008). Se ha demostrado que la acción del THC sobre células de glioma resulta en síntesis de ceramida y muerte celular, mientras que la misma sustancia brinda una acción protectora sobre los astrocitos contra el estrés oxidativo (Carracedo et al., 2004).

\section{Posible influencia de la cronobiología en la acción anticancerígena de los canabinoides $y$ endocanabinoides}

Los ritmos circadianos en los seres vivos gobiernan un gran número de funciones fisiológicas, incluyendo el 
ciclo sueño-despertar, la producción de hormonas, la formación y liberación de neurotransmisores y el ciclo celular, entre otros. Así, los ritmos biológicos juegan un papel importante tanto en la salud como en la enfermedad. Ésta es la razón por la cual en la actualidad se está haciendo un gran esfuerzo por conocer los parámetros de la cronofarmacología, que permitan una acción cronoterapéutica eficaz y potencialmente exenta de las complicaciones que trae el uso de fármacos en horarios establecidos sin tener en cuenta los ciclos circadianos. Estudios de varios agentes antineoplásicos han demostrado que cada uno posee un patrón de acción cronofarmacológica óptimo, de acuerdo con la fase del ciclo celular en la cual ejercen su acción citotóxica (Lévi, 2001; Lévi, 2002; Mormont and Lévi, 2003). Así, el 5-fluorouracilo (5-FU) presenta un pico máximo de acción citotóxica a las 4 p.m (Goldbeter et al., 2007), para pacientes con cáncer, cuya actividad es diurna, y, posiblemente, resultará diferente en pacientes con cáncer cuya actividad sea nocturna. Hasta el momento es poco lo que se ha hecho al respecto, considerando la acción antineoplásica de los endocanabinoides y canabinoides.

\section{Conclusiones}

La quimioterapia actual del cáncer, que utiliza fármacos como los agentes alquilantes, antimetabolitos, antibióticos antineoplásicos, cisplatino y sus derivados y alcaloides de la vinca, ha resultado efectiva en muchos de los tratamientos antineoplásicos en la especie canina. Sin embargo, son bien conocidos los efectos tóxicos de dichos tratamientos. La comunidad científica ofrece para un futuro mediano nuevas alternativas terapéuticas, basadas en el comportamiento de los canabinoides y endoconabinoides en el control de las enfermedades neoplásicas, teniendo en cuenta su alto grado de selectividad sobre células cancerosas; lo anterior mitiga muchos de los efectos adversos observados en los tratamientos tradicionales. Dicha actividad podría potenciarse teniendo en cuenta los criterios cronofarmacológicos que orientan los ritmos biológicos del paciente.

\section{Referencias}

Adams HR. 2001. Farmacología y terapéutica veterinaria. 10 ed. Acribia, Zaragosa, España, p 1141-1162.

Alberich Jorda M, Rayman N, Tas M, Verbakel SE, Battista $\mathrm{N}$, van Lom $\mathrm{K}$ et al. The peripheral cannabinoid receptor CB2, frequently expressed on AML blasts, either induces a neutrophilic differentiation block or confers abnormal migration properties in a ligand-dependent manner. Blood 2004; 104: 526-534.
Atwood BK and Mackei K. CB2: a cannabinoid receptor with an identity crisis. Br. J. Pharmacol. 2010; 160: 467-479.

Aurrecochea R Juan Carlos. Quimioterapia antineoplásica en animales domésticos. REDVET, 2008: Vol IX, No 2, Pag $1-30$.

Bifulco $M$ and Di Marzo V. Targeting the Endocannabinoid System in Cancer Therapy: A Call for Further Research. Nat Med. 2002; 8: 547-550.

Bifulco $\mathrm{M}$ et al. Control by Endogenous Cannabinoid System of Ras Encogen-Dependent Tumor Growth. FASEB J. 2001; 15: 2745-2747.

Bifulco M, Laezza C, Valenti M, Ligresti A, Portella G, Dim $\mathrm{V}$. A new strategy to block tumor growth by inhibiting endocannabinoid inactivation FASEB J, 2004; 18: 1606-1608.

Bifulco M., Laezza C., Gazzero P. And Pentimalli F. Endocannabinoids as emerging Suppressors of Angiogenesis and Tumor Invasion (Review). Oncology Reports, 2007; 17(4): 813-816.

Blázquez C., González-Feria L, Álvarez L, Hato A, Casanova ML and Guzmán M. Cannabinoids Inhibit the Vascular Endothelial Growth Factor Pathway in Gliomas. Cancer Res. 2004; 64: 5617-5623.

Bosier B, Muccioli GG, Hermans E and Lambert DM. Functionally selective cannabinoid receptor signalling: Therapeutic implications and opportunities. Biochem Pharmacol. 2010; 80: 1-12.

Bouaboula M. et al. Activation of Nitrogen-Activated Protein kinases by Stimulation of the Central Cannabinoid Receptor CB1. Biochem J. 1995; 312: 637-641.

Bracho AG. Oncología, Revista del colegio de médicos veterinarios el estado de Lara, 2011: Vol1, No1, Pag 15-19.

Calvelo RF. Mecanismos de acción antitumoral. Boletin oncológico, 2013, Pag 25-51. Tomado de www. Boloncol.com/index2.

Carracedo A, Geelen MJ, Diez M, Hanada K, Guzmán M, Velasco G. Ceramide sensitizes astrocytes to oxidative stress: protective role of cannabinoids. Biochem J 2004; 380: 435-440.

Contassot E, Wilmotte R, Tenan M, Belkouch MC, Schunuriger $\mathrm{V}$, de Trbolet $\mathrm{N}$ et al. Arachidonoylethanolamine induces apoptosis of human glioma cells through vanilloid receptor-1. J Neuropathol Exp Neurol, 2004; 63: 956-963.

De Lago E, Gustafsson SB, Fernández-Ruiz J, Nilson J, Jacobsson SO, Fowler CJ. Acyl-based anandamide uptake inhibitorscause rapid toxicity to C6 glioma cells at pharmacologically relevant concentrations. Neurochem. 2006; 99: 677-688.

De Morrow S., Glaser S. et al. Opposing Actions of Endocannabinoids on Cholangiocarcinoma Growth: Recruitment of Fas and Fas Ligand to Lipid Rafts. J Biol Chem, 2007; 282: 13098-13113.

De Petrocellis L., Melck D., Palmisano A., Bisogno T., Bifulco M, Di Marzo V. The Endogenous Cannabinoid Anandamide Inhibits Human Breast Cancer Cell 
Proliferation. Proc. Natl. Acad. Sci. USA. 1998; 95: 8375-8380.

Devane WA et al. Isolation and Structure of a Brain Constituent that Binds to the Cannabinoid Receptor. Science, 1992; 258: 946-1949.

Donadelli M. et al. Gemcitabine/cannabinoid combination triggers autophagy in pancreatic cancer cells through a ROSmediated mechanism. Cell Death Dis. 2011; 2: 152.

Elliott JW, Cripps P, Marrington AM, Grant A and Blackwood L. Epirubicin as part of a mult- agent chemotherapy protocol for canine lymphoma. Vet Comp Oncol. 2013; 11(3): 185-198.

Endsley MP, Aggarwal N, Isbell MA, Wheelock CE, Hammock BD, Falck JR et al Diverse roles of 2-araquidonoylglycerol in invasion of prostate carcinoma cells: Location, hydrolysis and 12-lipoxygenase metabolism. Int J Cancer, 2007; 121: 984-991.

Flygare J and Sander B. The Endocannabinoid system in Cancer: Potential Therapeutic Target? Seminars in Cancer Biology, 2008; 18: 176-189.

Fowler CJ, Gustafsson SB, Chung SC, Persson E, Jacobsson SOP and Bergh A. Targeting the endocannabinoid system for the treatment of cancer- a practical view. Curr Top Med Chem, 2010; 10: 814-827.

Galiegue S. et al Expression of Central and Peripheral cannabinoid Receptors in Human Inmune Tissues and Leukocytes Subpopulations. Eur J Biochem, 1995; 232: 54-61.

Gardner B, Zhu LX, Sharma S, Tashkin DP, Dubinett SM. Methanandamide increases COX-2 expression and tumor growth in murine lung cancer. FASEB J. 2003; 17: 2157-2159.

Giuffrida A. et al. Dopamine Activation of Endogenous Cannabinoid Signaling in Dorsal Striatum. Nature Neurosci, 1999; 2: 358-363.

Goldbeter A, Altinok A and Levi F. A cell cycle automaton model for probing circadian patterns of cancer drug delivery. Advanced Drug Delivery Reviews 2007; 59: 1036-1053.

Gómez del Pulgar T, De Ceballos ML, Guzmán M, Velasco G. Cannabinoids protect astrocytes from ceramide-induced apoptosis through the phosphatidylinositol 3-kinase/protein kinase B pathway. J Biol Chem, 2002; 277: 36527-36533.

Gómez del Pulgar T, Velasco G, Sánchez C, Haro A, Guzmán $M$. De novo synthetized ceramide is involved in cannabinoid-induced apoptosis. Biochem J, 2002; 363: 183-188.

Grimaldi C, Pisanti S, Laezza C, Malfitano AM, Di Marzo V. And Bifulco $M$ et al Anandamide Inhibits Adhesion and Migration of Breast Cancer Cells. Exp. Cell Res, 2006; 312: 363-373.

Grotenherm F. Farmacology of cannabinoids. Neuroendocrinol Letter. 2004; 1(2): 14-23.

Guindon J, Hohmann G. The endocannabinoid system and cancer: therapeutic implications. Br J Pharmacol. 2011; 163: 1447-1463.
Guzmán M. Cannabinoids: Potential Anticancer Agents. Nat. Rev Cancer. 2003; 3: 745-755.

Hart S, Fischer OM, Ulrich A. Cannabinoids induce cancer cell proliferation via tumor necrosis factor alpha-converting enzyme (TACE/ADAM17)- mediated transactivation of the epidermal growth factor receptor. Caner Res, 2004; 64: 1943-1950.

Herkenham M, Lynn AB, de Costa BR and Richfield EK. Cannabinoid Receptor Localization in Brain. Proc Natl Acad Sci USA 1990; 87: 1932-1936.

Herrera C Ibrahim, Thomas E Reginald, Fernández R Juan Carlos. Comprobación de la efectividad de dos esquemas terapéuticos en el tratamiento del tumor de Stiker en perros. REDVET. 2006; 7(10): 1-7.

Howlett AC. The CB1 Cannabinoid Receptor in the Brain Neurobiol. Dis. 1998; 5: 405-416.

Huang SM et al. An Endogenous Capsacin-like Substance with High Potency at Tecombinant and Native Vanilloid VR1 Receptor. Proc Natl Acad Sci, USA 2002; 99: 8400-8405.

Ishac EJ et al. Inhibition of Exocytotic Noradrenaline Release by Presynaptic Cannabnoid CB1 Receptors on PeripheralSympathetic Nerves. Br J Pharmacol, 1996; 118: 2023-2028.

Jorcano JL, Guzmán M et al. Inhibition of Tumor Angiogenesis by Cannabinoids. FASEB, 2003; 17: 529-531.

Jenny M, Schrocksnadel S, Uberal I F and Fuhcs D. The potential role of cannabinoids in modulating serotonergic signaling by their influence on tryptophan metabolism. Pharmaceuticals. 2010; 3: 2647-2660.

Katona I and Freund TF. Endocannabinoid signaling as a synaptic circuit breaker in neurological disease. Nature Med. 2008; 14: 923-930.

Klein TW, Newton C, Larsen K, Lu L, Perkins I, Nong L, et al. The cannabinoid system and immune modulation. J Leukoc Biol, 2003; 74: 486-489.

Kukanich B. Geriatric veterinary pharmacology. Vet Clin Small Anim, 2012; 42: 631-642.

Lévi F. Circadian chronotherapy for human cancers. Lancet Oncol 2001; 2: 307-315.

Lévi F. From circadian rithms to cancer chronotherapeutics. Chronobiol Int 2002; 19: 1-19.

Ligresti A, Bisogno T, Matias I, De Petrocellis L, Cascio MG, Cosenza $\vee$ et al. Possible Endocannabinoid Control of Colorectal Cancer Growth. Grastroenterology, 2003; 125: 677-687.

Liu J et al. Multiple pathways involved in the biosynthesis of anandamide. Neuropharmacology, 2008; 54: 1-7.

Maccarrone M, Lorenzon T, Bari M, Melino G, Finazzi-Agro A. Anandamide induces apoptosis in human cells via vanilloid receptors. Evidence for a protective role of cannabinoid receptors. J Biol Chem, 2000; 275: 31938-31945.

Maddison JE, Page SW, Church D. 2004. Farmacología clínica en pequeños animales. 1 ed. Intermédica, Buenos Aires, argentina, p 253-282. 
Mailleux P, Vanderhaeghen JJ. Distribution of Neuronal Cannabinoid Receptor in the Adult Rat Brain: A Comparative Receptor Binding Radioautography and In Situ Hybridazation Hystochemistry Neuroscience, 1992; 48: 655-668.

Malfitano AM et al. Update on the endocannabinoid system as an anticancer target. Expert Opin Ther Targets, 2011; 15: 297-308.

Mallat A, Teixeira-Clerc F, Deveaux V, Manin S and Coterztain $\mathrm{S}$. The endocannabinoide system as a key mediator during liver diseases: new insights and therapeutic openings. British J Pharmacol, 2011; 163: 1432-1440.

Marini $\mathrm{P}$ et al. Cannabinoid CB1 receptor elevation of intracellular calcium in neuroblastoma SH-SY5Y cells: interactions with muscarinic and $\delta$-opioid receptor. Bioch et Bioph Acta, 2009; 1793: 1289-1303.

Matas D, Juknat A, Pietr M, Klin Y, Vogel Z. Anandamide protects from low serum-induced apoptosis via its degradation to ethanolamine. J Biol Chem 2007; 282: 7885-7892.

Matsuda LA, Lolait SJ, Brownstein MJ, Young AC and Bonner TI. Structure of Cannabinoid Receptor as Functional Expression of the Coned cDNA. Nature, 1990; 346: 561-564.

Mayorga F and Cárdenas R. Endocannabinoids: A therapeutic option to cancer treatment. Vitae, 2009; 16(2): 259267.

Mechoulam R. et al. Identification of an Endogenous 2-monoglyceride, Present in Canine Gut, that Binds to the Cannabinoid Receptors. Biochem. Pharmacol, 1995; 50: 83-90.

Melck D, De Petrocellis L. et al. Supression of Nerve Growth Factor TrK receptors and Prolactin Receptors by Endocannabinoids Leads to Inhibition of Human Breast and Prostate Cancer Cell Proliferation. Endocrinology, 2000; 141(1): 118-126.

Meuten JD. 2002. Tumors in domestic animals. 4 ed. lowa State Press, lowa, United States of America, p 3-40.

Mimeault M, Pommery N, Wattez N, Bailly C, Henichart JP. Antiproliferative and apoptotic effects of anandamide in human prostatic cancer cells lines: implication of epidermal growth factor receptor down-regulation and ceramide production. Prostate, 2003; 56: 1-12.

Moore S Antony and Kitchell E Barbara. New chemotherapy agents in veterinary medicine. Vet Clin Small Anim, 2003; 33: 629-649.

Mormont MC, Lévi F. Cancer chronotherapy: principles, applications and perspectives. Cancer, 2003; 97: 155169.

Munro S., Thomas KL and Abu-Shaar M. Molecualr Caracterization of a Peripheral Receptor of Cannabinoids. Nature, 1993; 365: 614-618.

Munson AE et al. Antineoplasic Activity of Cannabinoids. J Nat Cancer Inst, 1975; 55: 597-602.

Nelson JR. et al. Systemic and Portal Hemodynamic Effects of anandamide. Am J. Physiol. Gastrointest. Liver Physiol, 2001; 280: 14-20.
Nithipatikom K, Endsley MP, Isbell MA, Falck JR, Iwamoto $Y$, Hillard CJ et al. 2-araquidonoylglycerol: a novel inhibitor of androgen-independent prostate cancer cell invasion. Cancer Res, 2004; 64: 8826-8830.

Nithipatikom K, Endsley MP, Isbell MA, Wheelock CE, Hammock BD, Campbell WB. A new class of inhibitors of 2-araquidonoylglycerol hydrolysis and invasion of prostate cancer cells. Biochem Biophys Res Commun, 2005; 332:1028-1033.

Nithipatikon K, Endsley MP, Isbell MA, Wheelock CE, Mammock BD, Campbell WB. A New Class of Inhibitors of 2-arachidonoylgycerol Hydrolysis and Invasion of Prostate Cancer Cells. Biochem. Biophys. Res Commun, 2005; 332: 1028-1033.

Ohdon S. Chronotherapeutic strategy: rhythm monitoring, manipulation and disruption. Adv Drug Deliv Rev, 2010; 62: 859-875.

Pacher P, Batkai S and Kunos G. The endocannabinoid system as an emerging target of pharmacotherapy. Pharmacol Rev, 2006; 58: 389-462.

Paolini M and Khanna C. Traslation of new cancer treatments from pet dogs to humans. Nat Rev Can, 2008; 8: 147156.

Pertwee RG. The diverse CB1 and CB2 receptor pharmacology of three plant cannabinoids: $\Delta^{9}$ - tetrahydrocannabinol, cannabidiol and $\Delta^{9}$ tetrahydrocannabivarin. $\mathrm{Br} J$ Pharmacol, 2008; 153: 1999-2015.

Pertwee RG. Emerging strategies for exploting cannabinoid receptor agonists as medicines. Br J Pharmacol, 2009; 156: $397-411$.

Portella G, Laezza C, Laccetti P, De Petrocellis L, Di Marzo $\mathrm{V}$, Bifulco M. Inhibitory effects of cannabinoid CB1 receptor stimulation on tumor growth and metastatic spreading: actions on signals involved in angiogenesis and metastasis. FASEB J. 2003; 17: 1771-1773.

Porter AC et al. Characterization of a Novel Endocannabinoid, Virodhamine, with Antagonist Activity at the CB1 Receptor. J Pharmacol Exp Ther, 2002; 30; 1020-1024.

Reagan RC, Kaplan MSW and Baley DB. Diagnostic evaluation and treatment recommendations for dogs with substage- a- high- grade multicentric lymphoma: result of a survey of a survey of veterinarians. Vet Comp Oncol, 2013; 4: 287-295.

Rodríguez U., Carrillo, E. y Soto, E. Canabinoides: Neurobiología y Usos Médicos. Elementos, 2005; 12(60):3-9.

Sánchez C et al. Delta-9-Tetrahydrocannabinol Inhibits Cell Cycle Progression in Human Breast Cancer Cells Through Cdc2 Regulation. Cancer Res, 2006; 66(13): 6615-6621.

Sarfaraz S, Afaq F, Adhami VM, Malik A, Mukhatar H. Cannabinoid receptor agonist-induced apoptosis of human prostate cancer cells LNCaP proceed through sustained activation of ERK1/2 leading to G1 cell cycle arrest. J Biol Chem, 2006; 281: 39480-39491.

Sarfaraz S, Afaq F, Adhami VM, Mukhtar H. Cannabinoid Receptors as Novel Target for the Treatment of Prostate Cancer. Cancer Res, 2005; 65: 1635-1641. 
Sarfaraz S, Adhami VM, Syed DN, Afaq F, Mukhtar H. Cannabinoids for cancer treatment: progress and promise. Cancer Res, 2008; 68: 339-342.

Sarnataro D, Pisanti S, Gazzerro P, Malfitano AM, Laezza $\mathrm{C}$, et al. The cannabinoid CB1 receptor antagonist rimonobant (RS141716A) inhibits human breast cancer cells proliferation through a lipid raft-mediated mechanism. Mol Pharmacol, 2006; 70: 1298-1306.

Smith TH, Sim-Selley LJ and Selley DE. Cannabinoid CB1 receptor- interacting proteins: novel targets for central nervous system drug discovery. Br J Pharmacol, 2010; 160: 454-466.

Smolensky MH, Peppas NA. Chronobiology, drug delivery and chronotherapeutics. Advanced Drug Delivery Reviews, 2007; 59: 828-851.

Souza CM, Toledo-Piza E, Amorin R, Barboza A, Tobias KM. Inflamatory mammary carcinoma in 12 dogs: clinical features, cyclooxigenase 2 expression and response to piroxicam treatment. Can Vet J, 2009; 50: 506-510.

Strieter RM. Masters of Angiogenesis. Nat Med, 2005; 11: 925-927.
Torres S. et al. A combined preclinical therapy of cannabinoids and temozoamide against glioma. Mol Cancer Ther, 2011; 10: 90-103.

Tsou K. et al. Inmunohistochemical Distribution of Cannabinoid CB1 Receptors in the Rat Cantral Nervous System Neuroscience, 1998; 83: 393-411.

Vail DM, Rodabargh HS Conder GA et al. Efficacy of injectable maropitant (cerenia) in a randomized clinical trial for prevention and treatment of cisplatin- induced emesis in dogs presented as veterinary patients. Vet Comp Oncol, 2007; 5: 38-46.

Van Meevenne S, Verhoeven PS, De Vos J, Gielen IMVL, Polis I and Van Ham LML. Comparison between symptomatic treatment and lomustina supplementation in 71 dogs with intracranial, space occupying lesions. Vet Cmp Oncol, 2012; 12(1): 67-77.

Velasco G, Sánchez C, Guzmán M. Towards the use of cannabinoids as antitumour agents. Nat Rev Can, 2012; 12(6): 436-444.

Withrow SJ and Vail DM. Oncología clínica de pequeños animales. 2007, 4 ed. Saunders, an inprint of elsevier. p 55. 